\title{
The role of viscoelasticity on heterogeneous stress fields at frictional interfaces
}

\author{
M. Radiguet* , D.S. Kammer, J.F. Molinari \\ Computational Solid Mechanics Laboratory (LSMS), Ecole Polytechnique Fédérale de Lausanne (EPFL), IIC-ENAC, Station 18, 1015 Lausanne, Switzerland
}

\section{A R T I C L E I N F O}

\section{Article history:}

Received 1 November 2013

Received in revised form 31 January 2014

Available online $\mathrm{xxxx}$

\section{Keywords:}

Frictional interface

Heterogeneous stresses

Viscoelasticity

Dynamic simulations

History effect

\begin{abstract}
A B S T R A C T
We investigate the evolution of heterogeneous stress states along frictional interfaces. Using finite-element simulations, we model the occurrence of precursory slip sequences on a deformable-deformable as well as a deformable-rigid interface between two solids. Every interface rupture creates a stress concentration at its arrest position and erases the stress concentrations produced by previous slip events. While the interface is sticking perfectly between two slip events, erased stress concentrations reappear and survive several cycles of ruptures. Such reestablished stress concentrations are smaller than they were before the rupture. We show that the decrease rate of these stress concentrations is exponential with respect to the number of subsequent events and that the bulk viscoelasticity is at the origin of this history effect. During a slip event, the friction tractions at the interface change almost instantaneously, which leads, between two ruptures, to a relaxation-resembling viscous effect that restores the stress concentration. We describe the decrease rate analytically and highlight that it is proportional to the ratio of the viscous over the instantaneous Young's moduli, and illustrate it by varying the material properties in our simulations.
\end{abstract}

(c) 2014 Elsevier Ltd. All rights reserved.

\section{Introduction}

Understanding the dynamic behavior of frictional interfaces is relevant for a broad range of applications in engineering, mechanics and geophysics. Despite longstanding research on friction, its dynamics is today still not well understood. For instances, the knowledge of the macroscopic frictional strength is essential to the design of new engineering applications. Nevertheless, the transition from sticking to sliding, which is closely related to the macroscopic friction coefficient, is still an only partially known phenomenon. The classic view considers that sliding initiates when the macroscopic shear force overcomes the macroscopic friction force, which is proportional to the normal loading. This simplistic picture does not

\footnotetext{
* Corresponding author. Tel.: +41 216932413.

E-mail address: mathilde.radiguet@epfl.ch (M. Radiguet).
}

account for the complexity of local slip occurring during the initiation of sliding. The transition from sticking to sliding was shown, in experiments, to be particularly interesting when the shear load of an interface between two blocks is applied by a pusher that is located near the interface (Rubinstein et al., 2007; Maegawa et al., 2010). In these conditions, the sliding initiation is a succession of local slip episodes that propagate at macroscopic stress levels much below the friction coefficient of the system. These precursors to global sliding initiate at the trailing edge, where the pusher is applied, and propagate along the interface until they stop before the other edge. Generally, each slip event propagates further than the previous rupture, until they reach the leading edge, which is considered to be global sliding. Numerical simulations using mass-spring models (1D (Maegawa et al., 2010; Amundsen et al., 2012) and 2D (Trømborg et al., 2011)) reproduced qualitatively this behavior and show the 
importance of asymmetric shear loading for the existence of such precursory slip. Other aspects of local slip observed in experiments (Rubinstein et al., 2004; Ben-David et al., 2010), such as a large range of rupture velocities, were also successfully reproduced by numerical simulations (Bar-Sinai et al., 2013; Kammer et al., 2012; Otsuki and Matsukawa, 2013).

The global sliding initiation is complex due to the presence of precursors, but the dynamics of these local slip events is complex in itself. Every precursor modifies durably the stress state of the interface (Rubinstein et al., 2007). Recursively, this means that every precursor propagates at an interface of highly heterogeneous stresses created by the history of previous ruptures. Furthermore, inhomogeneous stress states affect the propagation velocity of local slip events (Ben-David et al., 2010; Kammer et al., 2012), which results also in modified stress states after the current rupture and hence affects the succeeding slip event as well.

In addition to local dynamics influencing the stress state of the interface, it is suspected that a bimaterial effect contributes to the complexity of the problem. Most experiments were conducted on a non-symmetric interface, with a thin slider in contact with a much thicker base block (Rubinstein et al., 2007; Ben-David et al., 2010; Maegawa et al., 2010). The difference in thickness results in different effective stiffnesses, making the interface bimaterial. Ruptures at bimaterial interfaces are known for their richness of complex mechanics (Coker et al., 2003; Lykotrafitis and Rosakis, 2006) and to date no experiments of similar material interfaces have been conducted to compare with the bimaterial system of Rubinstein et al. (2007). In all numerical studies of the transition from sticking to sliding, this bi-material effect has been approximated by a deformable-rigid interface (Maegawa et al., 2010; Amundsen et al., 2012; Trømborg et al., 2011; Kammer et al., 2012; Bar-Sinai et al., 2013; Otsuki and Matsukawa, 2013).

In recent numerical simulations, Radiguet et al. (2013) revealed that stress concentrations created by the arrest of precursory slip survive several succeeding interface ruptures. Using an analytic approach, they proposed that the viscous property of the bulk material is at the origin of this history effect. In this paper, we confirm the proposed analytic model with new numerical results by varying the material properties. Further, for the first time, we present results from simulations of precursory slip at a deformable-deformable interface, and analyze the effect of the bimaterial interface on the survival of stress concentrations.

\section{Simulation setup}

\subsection{Loading characteristics}

The simulated system (Fig. 1) consists of a two-dimensional rectangular top plate (slider), of length $L_{t}=0.2 \mathrm{~m}$ and height $H_{t}=0.1 \mathrm{~m}$, in plane-stress approximation. The slider is in contact with a two-dimensional rectangular bottom plate (base) of dimensions $L_{b}=0.4 \mathrm{~m}$ and $H_{b}=0.05 \mathrm{~m}$. The base, is either approximated as a rigid body, as it has been done in previous numerical studies,

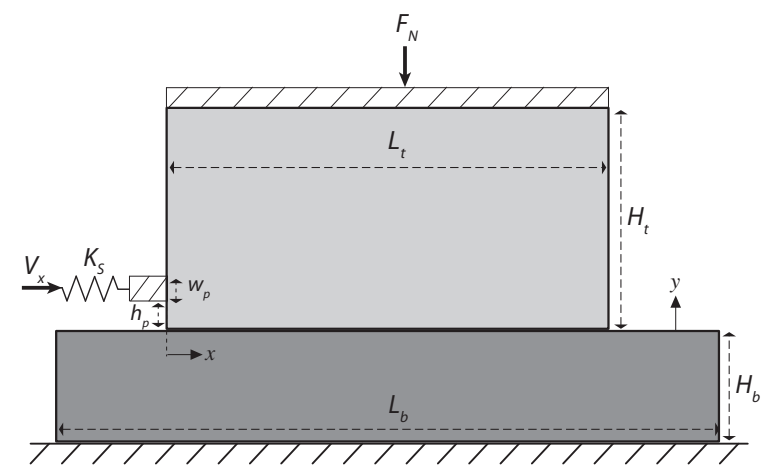

Fig. 1. Simulation setup. The upper plate (slider) of dimensions $L_{t} \times H_{t}$ is viscoelastic in plane-stress approximation. This plate is in contact with a bottom plate (base) of dimensions $L_{b} \times H_{b}$, which can be either rigid or deformable (plane-stress or plane-strain). The origin of the reference axis is the initial position of the lower left corner of the upper plate.

or as a deformable viscoelastic solid in plane-strain or plane-stress approximation. If the base is rigid, its dimensions have no influence on the system. The bottom edge of the block is fixed in the $\mathrm{x}$ and $\mathrm{y}$ direction. On the lateral sides of the base, no displacements or forces are imposed. A constant normal load $F_{N}$ is applied to the top surface of the slider. The shear load is applied via a spring of stiffness $K_{S}$ and a rigid pusher to the left side of the top plate. A constant loading velocity $V_{x}$ is imposed and the macroscopic shear force $F_{S}$ is measured at the spring of the pusher. The values given in Table 1 are equivalent to the parameters used in the simulations of Radiguet et al. (2013), corresponding to an out-of-plane thickness of $6 \mathrm{~mm}$. The pusher of width $w_{p}$ is located at a distance $h_{p}$ from the interface, which is a lower position than was used in Radiguet et al. (2013). We use the finite-element code Akantu (2013) to simulate the continuum of the two solids. The formulation is discretized in time using an explicit Newmark- $\beta$ scheme. The plates are discretized using quadrangular elements, and the element size is $1 \mathrm{~mm}$, which is fine enough to ensure mesh convergence.

\subsection{Friction law and interface description}

Contact and friction at the interface are modeled using the traction-at-split-nodes technique (Andrews, 1999). The boundary conditions at the interface can be described by:

- For $\dot{u}_{x}(x, y=0) \neq 0$ (during slip):

$$
\sigma_{x y}(x, y=0, t)=\tau^{s}(x, t) \quad \& \quad\left[\left|u_{y}(x, y=0, t)\right|\right] \geqslant 0
$$

- For $\dot{u}_{x}(x, y=0)=0$ (during stick):

$$
\sigma_{x y}(x, y=0, t)<\tau^{s}(x, t) \quad \& \quad\left[\left|u_{y}(x, y=0, t)\right|\right]=0
$$

\footnotetext{
${ }^{1}$ Simulations performed with a finer mesh (element size of $0.5 \mathrm{~mm}$ ) show no significant differences in the global behavior, and in the evolution of the amplitude of stress concentrations compared to the mesh size used in this study.
} 
Table 1

List of material, friction, geometry and loading parameters.

\begin{tabular}{|c|c|c|c|}
\hline Parameter & Units & Reference & Variations \\
\hline \multicolumn{4}{|l|}{ Dimensions } \\
\hline Slider length $L_{t}$ & $\mathrm{~m}$ & 0.2 & \\
\hline Slider height $H_{t}$ & $\mathrm{~m}$ & 0.1 & \\
\hline Base length $L_{b}$ & $\mathrm{~m}$ & 0.4 & \\
\hline Base height $H_{b}$ & $\mathrm{~m}$ & 0.05 & \\
\hline Base characteristics & - & Rigid & $\begin{array}{l}\text { pl.-strain } \\
\text { pl.-stress }\end{array}$ \\
\hline \multicolumn{4}{|l|}{ Material } \\
\hline Viscous Young's modulus $E_{0}$ & GPa & 3 & $1.5-4.5$ \\
\hline Static Young's modulus $E_{\infty}$ & GPa & 2.6 & \\
\hline Viscosity $\eta$ & MPa.s & 5 & $5-500$ \\
\hline Poisson's ratio $v$ & - & 0.37 & \\
\hline Density $\rho$ & $\begin{array}{l}\mathrm{kg} / \\
\mathrm{m}^{3}\end{array}$ & 1200 & \\
\hline $\begin{array}{l}\text { Longitudinal wave speed } c_{L} \text { (pl.- } \\
\text { stress) }\end{array}$ & $\mathrm{m} / \mathrm{s}$ & 2325 & \\
\hline Shear wave speed $c_{S}$ & $\mathrm{~m} / \mathrm{s}$ & 1305 & \\
\hline \multicolumn{4}{|l|}{ Friction } \\
\hline Static friction coefficient $\mu_{s}$ & - & 0.7 & \\
\hline Kinetic friction coefficient $\mu_{k}$ & - & 0.45 & \\
\hline Slip weakening length $d_{c}$ & $\mu \mathrm{m}$ & 1 & \\
\hline Regularization time scale $t^{*}$ & $\mu \mathrm{s}$ & 50 & \\
\hline \multicolumn{4}{|l|}{ Boundary conditions } \\
\hline Horizontal loading velocity $V_{x}$ & $\mathrm{~mm} / \mathrm{s}$ & 2.5 & \\
\hline Normal load $F_{N}$ & $\begin{array}{l}\mathrm{MN} / \\
\mathrm{m}\end{array}$ & 1.042 & \\
\hline $\begin{array}{l}\text { Stiffness of horizontal loading spring } \\
K_{S}\end{array}$ & $\begin{array}{l}\mathrm{GN} / \\
\mathrm{m}^{2}\end{array}$ & 1 & \\
\hline Pusher width $w_{p}$ & $\mathrm{~mm}$ & 10 & \\
\hline $\begin{array}{l}\text { Distance between pusher and } \\
\text { interface } h_{p}\end{array}$ & $\mathrm{~mm}$ & 6 & \\
\hline
\end{tabular}

where $\sigma_{y y}(x, y=0, t)$ and $\sigma_{x y}(x, y=0, t)$ are the normal and tangential stresses at the interface, $\tau^{s}(x, t)$ is the frictional strength of the interface, $\dot{u}_{x}(x, y=0)$ is the slip velocity, and $\left[\left|u_{y}(x, y=0, t)\right|\right]$ is the interface gap.

The frictional strength is given by $\tau^{s}(x)=$ $\mu(l) \sigma_{y y}(x, y=0)$, where $\mu(l)$ is the friction coefficient. We use a slip weakening friction law, which is described by:

$$
\mu(l)= \begin{cases}\mu_{s}-\left(\mu_{s}-\mu_{k}\right) l / d_{c} & l<d_{c} \\ \mu_{k} & l \geqslant d_{c}\end{cases}
$$

with the characteristic weakening length $d_{c}$, and the static and dynamic friction coefficients $\mu_{s}$ and $\mu_{k}$. Applied values are given in Table 1 . The local slip $l$ increases continuously during sliding and is reset to zero once the interface sticks again $\left(l=0\right.$ when $\left.\dot{u}_{x}(x, y=0)=0\right){ }^{2}$

In addition to the linear slip weakening friction law, the frictional strength $\tau^{s}$ is regularized using a simplified form of the Prakash-Clifton law (Cochard and Rice, 2000), where the evolution of $\tau^{s}$ evolves as: $\dot{\tau}^{s}(t)=-1 / t^{*}$ $\left[\tau^{s}(t)-\mu(l) \sigma_{y y}(x, y=0, t)\right]$. This formulation was used

\footnotetext{
${ }^{2}$ The slip weakening law prescribes an instantaneous recovery of the static friction coefficient when slip stops. The regularization of the frictional strength introduces a small delay in the recovery of the frictional strength. The ageing of frictional interfaces after slip, as experimentally observed (Ben-David et al., 2010), is usually described by a logarithmic ageing law which is beyond the scope of this paper.
}

previously in numerical simulations (e.g. Bartolomeo et al., 2012; DeDontney et al., 2012; Radiguet et al., 2013). This regularization was initially proposed to account for the delay observed experimentally in the frictional strength as a result of sudden normal stress variation (Prakash and Clifton, 1993). The delay is controlled by a characteristic time $t^{*}$, which is small compared to the typical duration of slip events $(\approx 1 \mathrm{~ms})$. The influence of a small $t^{*}$ has been shown to be negligible for a single interface rupture (Kammer et al., 2014).

\subsection{Viscoelastic material}

In the experiments of precursory slip, a transparent material was used for the specimens in order to allow the measurement of the real contact area using a laser sheet technique. The chosen material, poly (methyl methacrylate), also known as PMMA, is known to be viscoelastic. In our simulations, we model the viscoelasticity of the bulk material using the standard linear solid model (Zener, 1948) applied only on the deviatoric part of the strain tensor. The spherical part of the strain tensor is often elastic for polymers. Governed by this constitutive law, the solid evolves from an instantaneous $E_{0}=E_{\infty}+E_{v}$ to the static $E_{\infty}$ Young's modulus over a single relaxation time scale, given by $t_{v}=\eta / E_{v}$, where $E_{v}$ is the viscous Young's modulus and $\eta$ is the viscosity.

Hooke's law defines the following relation between the strain $\epsilon_{i j}$ and the stress $\sigma_{i j}$ tensors:

$\sigma_{i j}=K \epsilon_{k k} \delta_{i j}+2 G\left(\epsilon_{i j}-\frac{1}{3} \epsilon_{k k} \delta_{i j}\right)$

where $K$ is the bulk modulus and $G$ the shear modulus. The first term in Eq. (2) corresponds to the spherical part of the stress tensor, and the second term to the deviatoric part.

As the viscosity is only applied to the deviatoric part of the stress tensor, we introduce the following relaxation function for the shear modulus $G(t)=G_{\infty}+G_{v} \exp \left(-t / t_{v}\right)$ and distinguish between the infinite $\left(K_{\infty}, G_{\infty}\right)$ and the viscous $\left(K_{v}, G_{v}\right)$ moduli. Considering an initial state of equilibrium $\sigma_{i j}\left(t_{0}\right)$ at $t_{0}$, an instantaneous perturbation in the strain tensor $\Delta \epsilon_{i j}$ results in the following evolution of the stress tensor:

$\sigma_{i j}(t)=\sigma_{i j}\left(t_{0}\right)+K_{\infty} \Delta \epsilon_{k k} \delta_{i j}+2 G(t)\left(\Delta \epsilon_{i j}-\frac{1}{3} \Delta \epsilon_{k k} \delta_{i j}\right)$

If the perturbation occurs in the shear component of the strain tensor only, $\Delta \epsilon=\Delta \epsilon_{x y}$, Eq. (3) becomes:

$\sigma_{x y}(t)=\sigma_{x y}\left(t_{0}\right)+2\left[G_{\infty}+G_{v} \exp \left(-\frac{t-t_{0}}{t_{v}}\right)\right] \Delta \epsilon_{x y}$

Introducing the Young's moduli and Poisson's ratio $v$, we get the following relations:

$\sigma_{x y}(t)=\sigma_{x y}\left(t_{0}\right)+V\left[E_{\infty}+E_{v} \exp \left(-\frac{t-t_{0}}{t_{v}}\right)\right] \Delta \epsilon_{x y}$

with $V=1 /(1+v)$ for plane-strain and $V=(1+2 v) /$ $(1+v)$ for plane-stress approximation. The values for the material properties are given in Table 1 . While the values for $E_{\infty}$ and $E_{v}$ represent well, in the reference case, the 
properties of PMMA (Ciccotti and Mulargia, 2004), $\eta$ is considerably smaller than the physical value. Nevertheless, the choice of $\eta$ for the reference case was done carefully, ensuring that the characteristic relaxation time $t_{v}(1)$ is sufficiently large such that variations in $E$ are negligible during a single slip event, and (2) that the ratio between $t_{v}$ and the inter-event time is similar between simulations and experiments. The imposed push velocity was therefore chosen accordingly.

In the following, we focus on the stresses at the interface. Therefore, we simplify the notation by introducing the interface shear stress $\tau=\sigma_{x y}(x, y=0)$ and normal stress $\sigma=\sigma_{y y}(x, y=0)$.

\section{Global behavior of the system}

In experiments (Rubinstein et al., 2007; Maegawa et al., 2010), the side-pusher set-up leads to precursory slip before macroscopic sliding. Generally, each precursor propagates further than the previous event did. Once a precursor propagates over the entire interface, a stick-slip regime takes place, in which the interface is mostly sticking and slip events repeatedly appear.

Our simulations reproduce well this behavior, as can be seen in Figs. 2 and 3. These figures present simulations performed in the reference case, in which the base is rigid. In Fig. 2, slip regions are shown in color, starting at $t=0.1 \mathrm{~s}$. The slip events always initiate at the trailing edge $(x=0 \mathrm{~m})$. There are 18 precursors before global sliding. The last precursor stops just before the right edge. The macroscopic response of the simulated system is shown in Fig. 3. The ratio between the measured shear load $F_{S}$ and the imposed normal load $F_{N}$ increases linearly in time until small jumps appear. These stress drops are caused by the released stresses at the interface due to precursors. The color of a stress drop matches with the corresponding precursor in Fig. 2. The macroscopic friction coefficient can be estimated by the peak value of the first slip event that

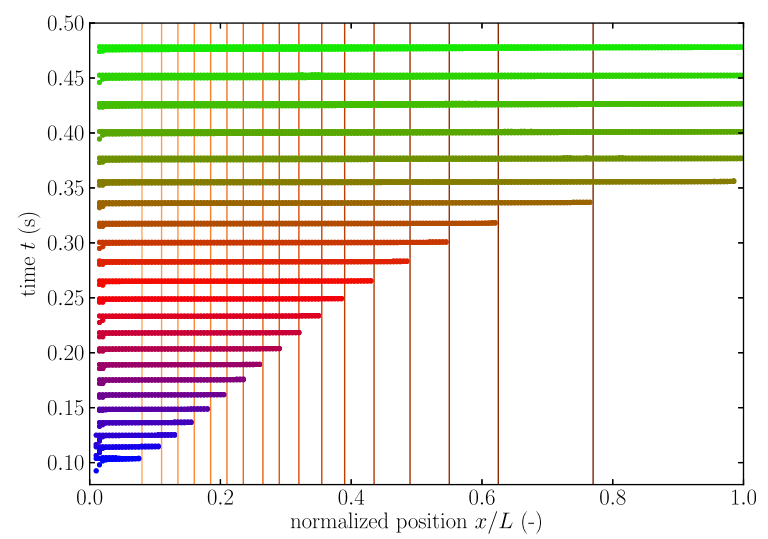

Fig. 2. Slipping region along the interface as a function of time. Colors from blue to green represent successive slip events of increasing lengths. The same color code refers to similar slip events in Figs. 3 and 5. Vertical lines are the locations $\left(x_{i}\right)$ of the stress concentrations created by each precursor arrest. (For interpretation of the references to colour in this figure caption, the reader is referred to the web version of this article.)

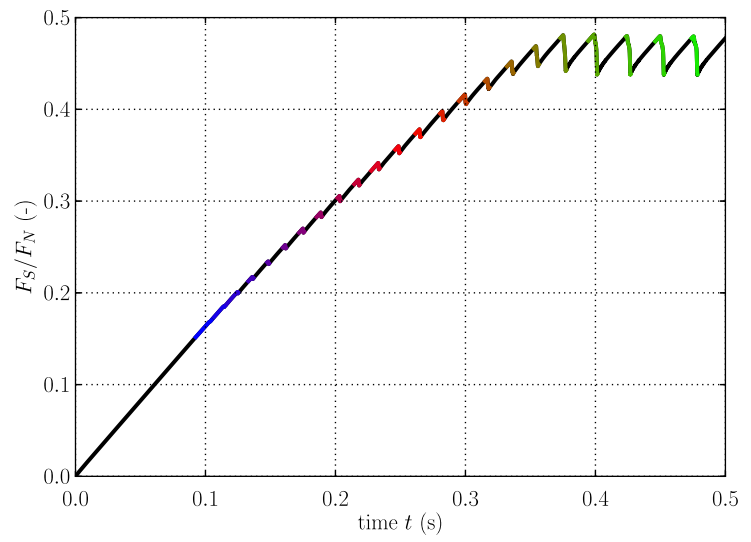

Fig. 3. Time evolution of the ratio of the global shear load $F_{S}$ over the global normal load $F_{N}$. Sections highlighted in colors represent the occurrence time of the slip events at the interface, and correspond to drops in $F_{S}$. The same color code as in Fig. 2 is used to identify slip events. (For interpretation of the references to colour in this figure caption, the reader is referred to the web version of this article.)

crosses the entire interface, and is here approximately $\mu_{m}=0.48$. The reference case is close to the simulations presented in Radiguet et al. (2013), the two differences being that the corners of the upper plate are not rounded and the pusher is located closer to the interface in this study. The use of non-rounded corners does not change the overall behavior. It only modifies the initiation of slip, because local stresses near the trailing edge, where slip initiates, are slightly different. This effect, in addition to the lower location of the pusher in this study compared to Radiguet et al. (2013), causes the present simulation to generate more precursor events before global sliding (18 instead of 11).

\section{Evolution of the stress concentrations}

\subsection{Stress evolution between successive slip events, in stick state.}

In this section, the effect of arrested slip events on the stress state of the interface is investigated. The propagation of a given slip event leaves the interface with a stress level close to $\tau=\mu_{k} \sigma$ behind the rupture tip, and a stress concentration exists at the tip of the arrested rupture (blue line in Fig. 4). Just after the arrest of slip, the interface is sticking and the evolution of shear stresses before the following event is shown as the lines from blue to red in Fig. 4). Just before the following slip event (red line), the interface is characterized by the existence of several stress concentrations: one is located at the tip of the previous event propagation, and its amplitude decreased during the stick state. The other stress concentrations reappeared during the stick phase, and are located at the arrest locations of previous precursors of shorter lengths.

To analyze the evolution of the stress concentration with successive ruptures, we focus on the stress state preceeding each rupture (equivalent to the red line in Fig. 4 for each precursor). This stress state is relevant because it was 


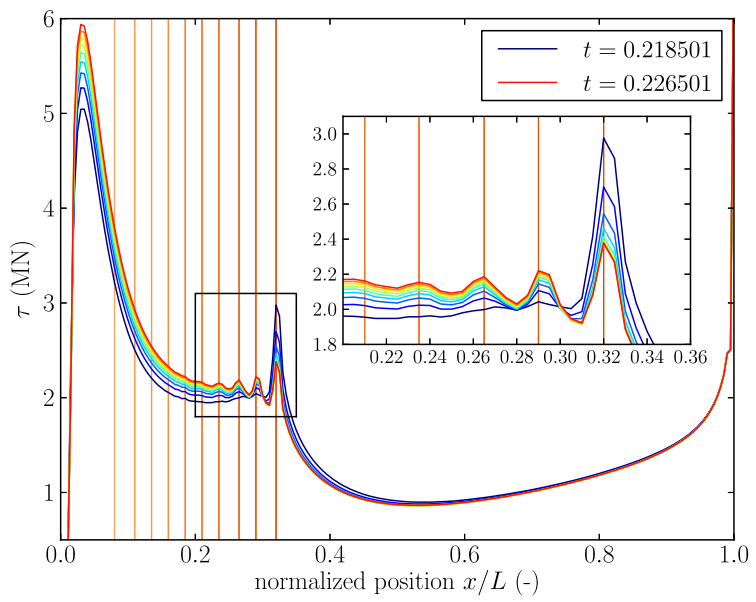

Fig. 4. Evolution of interface shear stresses between slip events ten and eleven. The system evolves from the blue line just after event ten to the red line just before event eleven. During this time, the relaxation of the bulk leads to the reappearance of stress concentrations at the interface. These stress concentration are located at the tip of the previous rupture arrest. The vertical lines mark the arrest location of previous precursors. Note that the red line corresponds to the bottom line in Fig. 5(b). (For interpretation of the references to colour in this figure caption, the reader is referred to the web version of this article.)

shown to influence the rupture propagation, and in particular the velocity of the rupture front (Ben-David et al., 2010). To characterize this stress state, we introduce a normalized quantity describing the amplitude of a stress concentration. In a second time, we propose a simple model to represent the time evolution of shear stress at the interface, as a function of the loading rate and the viscoelastic relaxation. This model allows us to relate the amplitude of stress concentrations between successive states of stress at the interface.

\subsection{Definition of the stress concentrations}

The stress concentration defined in Radiguet et al. (2013) were studied for the stress ratio $\tau / \sigma$, which illustrates well the evolution of the interface with respect to its strength. However, it was shown that the survival of these stress concentrations is adequately described by the shear tractions $\tau$ when changes in the contact pressure $\sigma$ are neglected. Therefore, we here focus our attention to stress concentrations only in the shear tractions $\tau$. As observed in Fig. 4, precursor $i$ creates a stress concentration at its arrest position. We measure this stress concentration $\Delta_{i}\left(\tau_{n}\right)$ at different times $t_{n}^{-}$just before the slip initiation of the following precursor $n$ : $\tau_{n}(x)=\tau\left(x, t_{n}^{-}\right)$. $\Delta_{i}\left(\tau_{n}\right)$ is defined starting from time $t_{i+1}$ (i.e. for $n>i$ ). The stress concentration is the difference in shear stress between the positions $x_{i}$ and $x_{i}^{\prime}$, where $x_{i}$ and $x_{i}^{\prime}$ are defined at time $t_{i+1}$ and kept constant afterward. $x_{i}$ is the local maximum in $\tau_{i+1}(x)$ around the position of the $i^{\text {th }}$ precursor arrest, and $x_{i}$ ' is the local minimum in $\tau_{i+1}(x)$ between $x_{i-1}$ and $x_{i}$.

$\Delta_{i}\left(\tau_{n}\right)=\tau_{n}\left(x_{i}\right)-\tau_{n}\left(x_{i}^{\prime}\right)$ for $n>i$
Fig. 5(b) represents graphically the value of $\Delta_{i}\left(\tau_{n}\right)$. The evolution of the stress concentration created by a given precursor $i$ can be analyzed for successive ruptures (i.e. for increasing values of $n$ ). To compare the stress concentrations created by different precursors (different positions $x_{i}$ ), we define the normalized amplitude of stress concentration $A_{i}^{n}$ as:

$A_{i}^{n}=\frac{\Delta_{i}\left(\tau_{n}\right)-\Delta_{i}^{r e f}}{\Delta_{i}\left(\tau_{i+1}\right)-\Delta_{i}^{r e f}}$ for $n>i$

$A_{i}^{n}$ is normalized such that its initial (maximum) value equals $1 . \Delta_{i}^{\text {ref }}$ corresponds to the reference level of difference in $\tau$ between the two positions $x_{i}$ and $x_{i}^{\prime}$, which is due to the loading configuration. It is defined as the time $t_{r}$ such that $\frac{\Delta_{i}\left(\tau_{r}\right)-\Delta_{i}\left(\tau_{r-1}\right)}{\Delta_{i}\left(\tau_{r}\right)}<1 \%$, and at the latest at the time of the second global sliding event.

The stress state of the interface just before each rupture (for all times $t_{n}$ ) is shown in Fig. 5, for the reference case in which the base is rigid. Lines are shifted along the $y$-axis to allow a better comparison of the evolutions of stress concentrations. The zoom (Fig. 5(b)) focuses on the stress concentration in $x_{10}$. The decreasing amplitude of the stress concentration with time is observed at all the positions $x_{i}$. Variations in the normal stress are less important (Fig. 5(c) and (d)).

The evolution of $A_{i}^{n}$ with the propagation of successive ruptures is presented in Fig. 6. At each position $x_{i}$ (different color line), $A_{i}^{n}$ decreases with a similar exponential decrease rate. Most curves collapse on a narrow range, and three deviate from the general tendency. These three curves correspond to the first precursors arrest locations. In that region, located close to the edge of the system, the slope of the stress decreases with time because of the loading, as can be seen in Fig. 5(a) around position $x / L=0.1$. For that reason, the reference $\Delta_{i}^{r e f}$ does not appropriately represent the reference level of stress concentration, and the evolution of $A_{i}^{n}$ varies from the global behavior. The mean evolution of $A_{i}^{n}$ is evaluated with and without taking into account the three positions that strongly deviates from the general behavior (respectively open and filled black dots and associated error bars). We observe that excluding the first three positions, the evolution of $A_{i}^{n}$ can be described with narrow error bars.

\subsection{Evolution of stresses in the system}

The evolution of stresses with time at the location $x_{10}$ (zoom in Fig. 5(b)) is shown in Fig. 7(a). The arrest of precursor 10 close to this position leads to an increase in $\tau$ (from the red to the blue dot). Then, successive slip events will propagate at that location. Each of them leads to an increase in $\tau$ up to the value of $\tau^{s}$ (dotted line) where slip initiates. A drop in $\tau$ is observed during the slip. When slip stops, $\tau^{s}$ recovers its static value, and $\tau$ evolves as described below.

The evolution of $\tau$ after the propagation of a slip event can be described by accounting for (1) the amplitude of the drop in shear stress caused by slip, (2) the effect of the viscoelastic relaxation and (3) the shear loading $p_{\tau}(x)$. 

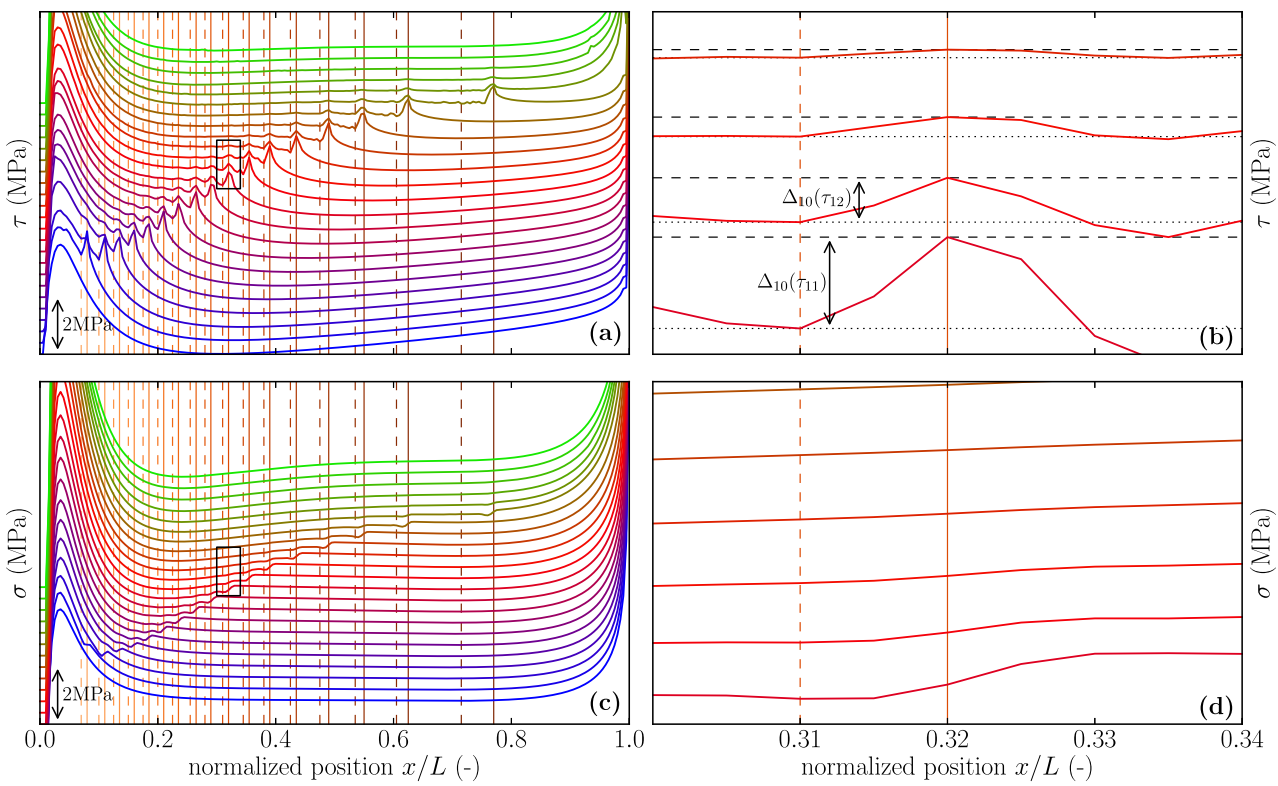

Fig. 5. Stresses at the interface just before each rupture. Each successive line is shifted upward along the $y$-axis to allow a better visualization of the evolution of stresses. The solid vertical lines are the positions $x_{i}$ and the dashed vertical lines the positions $x_{i}^{\prime}$. (a) Shear stress $\tau$ along the complete interface (b) Zoom on the arrest location of precursor 10. The rectangle in (a) shows the area of the zoom. The stress concentrations created by this precursor are shown by double arrows. (c) Normal stress $\sigma$ along the complete interface. (d) Zoom on the arrest location of precursor 10. The rectangle in (c) shows the area of the zoom.

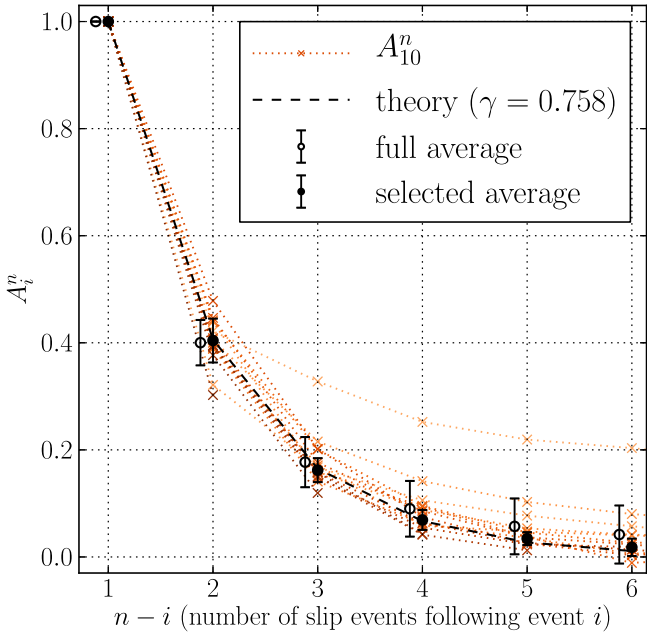

Fig. 6. Evolution of the normalized amplitude of stress concentration $A_{i}^{n}$ as a function of the number of successive ruptures following event $i$. Each line represents the evolution of the stress concentration at a given position $x_{i}$ along the interface, which was created by the slip event $i$. The open black circles correspond to the mean behavior of the system, with errors bars corresponding to one standard deviation. These dots are slightly shifted along the $x$-axis for clarity. The filled black circles represent the mean behavior of the system excluding the first three positions $x_{i}$ that strongly deviate from the mean behavior, due to their proximity to the trailing edge.

Assuming that complete relaxation has time to occur between slip events, the shear traction before event $n+1$ can be expressed as a function of the stress state of the system before the preceding event $n$ as (see Appendix A for details):

$$
\tau_{n+1}(x)=\mu_{k} \sigma_{n}(x)+\gamma \frac{E_{v}}{E_{0}}\left[\tau_{n}(x)-\mu_{k} \sigma_{n}(x)\right]+p_{\tau}(x)\left(t_{n+1}-t_{n}\right) .
$$

Volume changes in the system are neglected, so no viscous effect occurs on $\sigma$. Since the positions $x_{i}$ and $x_{i}^{\prime}$ are close, we can approximate $\sigma_{n}\left(x_{i}\right) \approx \sigma_{n}\left(x_{i}^{\prime}\right)$ (see Fig. 5(b)). Then, considering that the time between two successive slip events is almost constant for a given simulation, we can thus write (see Appendix B):

$A_{i}^{n}=\left(\gamma \frac{E_{v}}{E_{0}}\right)^{n-i-1}$ for $n>i$

This equation describes the decrease rate of $A_{i}^{n}$ for successive ruptures. It is controlled by the ratio of viscous over instantaneous Young's moduli, and the parameter $\gamma$, which accounts for the non-instantaneousness of the drop of shear stress during slip. This parameter $\gamma$ is obtained by best fit in Fig. 6, and gives a value of $\gamma=0.758$. The theoretical curve obtained with this $\gamma$ is plotted as a dotted line in Fig. 6, and describes well the mean decrease rates of the stress concentrations.

\section{Influence of viscoelastic parameters on the stress concentrations}

The relation given in Eq. (9) shows that the decrease rate of the stress concentrations is governed by the properties of the viscoelastic bulk material. In order to confirm this proposition, we conducted several simulations with varying material properties. The results are presented as the average behavior of the stress concentrations in Fig. 8 (points with error bars). 

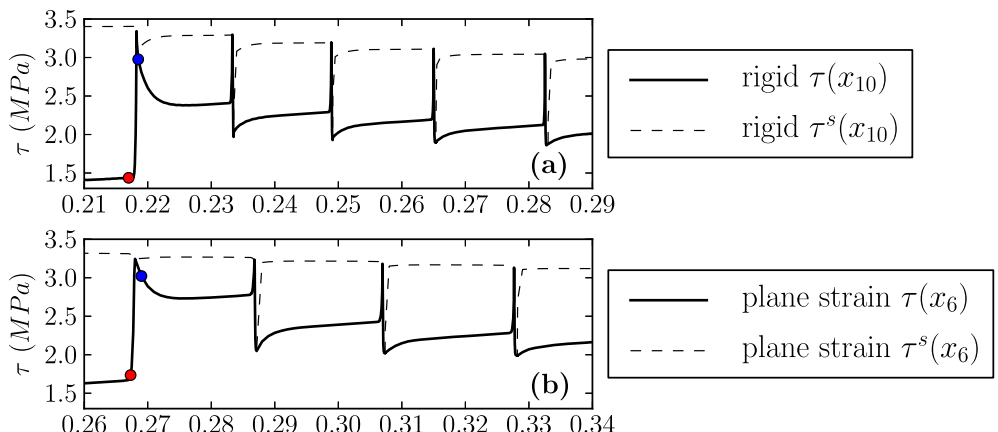

$\begin{array}{llllllllll}0.26 & 0.27 & 0.28 & 0.29 & 0.30 & 0.31 & 0.32 & 0.33 & 0.34\end{array}$

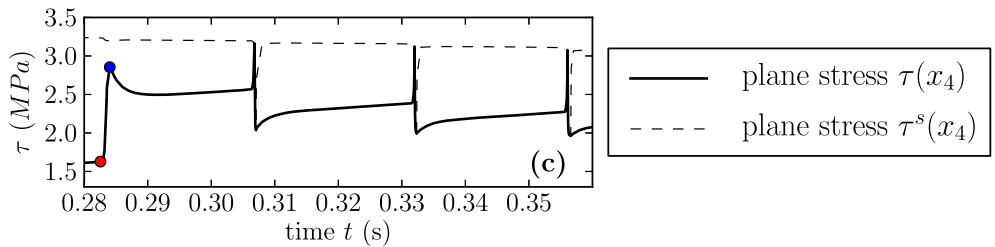

Fig. 7. Time evolution of $\tau$ (solid line) and shear strength (dashed line) on the interface for the closest stress concentration to the position $x / L=0.3$, for three different bases. The red dot marks the initial shear traction level $\tau\left(x_{i}, t_{i}^{-}\right)$, before the creation of the stress concentration in $x_{i}$. The blue dot marks $\tau\left(x_{i}, t_{i}^{+}\right)$, the value of $\tau$ just after event $i$, at the creation of the stress concentration, before the system relaxes. (a) Reference case, the base is rigid. The precursor 10 stops around $x / L=0.3$. (b) The base is deformable, in plane-strain approximation. The precursor 6 stops close to $x / L=0.3$. (c) The base is deformable, in plane-stress approximation. The precursor 4 stops close to $x / L=0.3$. (For interpretation of the references to colour in this figure caption, the reader is referred to the web version of this article.)

Qualitatively, the results are in a good agreement with the proposed theory given in Eq. (9). Smaller viscous Young's Moduli cause a higher decrease rate of the stress concentrations and thus shorten the survival of stress concentrations.

The influence of the viscosity $\eta$ on the decrease rate of stress concentrations is more complex. Generally, a larger $\eta$ results in a higher decrease rate for the first succeeding slip events $(n-i<2)$, but changes to a lower rate for later interface ruptures $(n-i>2)$. This behavior cannot be described by the proposed theory, because the assumption of complete relaxation between slip events is not satisfied anymore. Recall that the relaxation time is $t_{v}=\eta / E_{v}$. Larger $\eta$ result in longer relaxation times. If this is longer than the inter-event time, a slip front's viscous effect will influence the stress concentration even after the next rupture already propagated.

For all the simulations presented in this paper, the inter-event times are in the range of $10-30 \mathrm{~ms}$. In the reference case of the previous section, the relaxation time is $t_{v}=1.7 \mathrm{~ms}$, and thus it is reasonable to consider that complete relaxation occurs.

For cases presented in Fig. 8(a), where $E_{v}$ is varied, complete relaxation occurs in each case as well, because $t_{v}$ varies from $1.1 \mathrm{~ms}$ (for $E_{v}=4.5 \mathrm{GPa}$ ) to $3.3 \mathrm{~ms}$ (for $E_{v}=1.5 \mathrm{GPa}$ ). Therefore, in each case, the relaxation time is much smaller than the average inter-event time $t_{v} \ll\langle\Delta t\rangle$. The differences in the decrease rate of stress concentrations are thus only related to the changes in $E_{v}$ and the simplified theory given with in Eq. (9) is sufficient to describe the survival of stress concentrations (see dashed lines in Fig. 8(a)).

However, the material properties applied in the simulations presented in Fig. 8(b) have the following relaxation times: $t_{v}=7.7 \mathrm{~ms}$ (for $\eta=20.0 \mathrm{MPa} \mathrm{s}$ ) and $t_{v}=13.3 \mathrm{~ms}$ (for $\eta=40.0 \mathrm{MPa}$ ). As these times are non-negligible compared to the average inter-event times, the simplified theory cannot be applied. A more general approach, based on the same evolution of shear stresses at the interface (Eq. (A.4)), is derived in Appendix C. Considering complete relaxation within a period of two inter-event times, a recursive approach leads for $n>i+1$ to the following relation:

$$
\begin{aligned}
A_{i}^{n} & =\gamma \frac{E_{v}}{E_{0}}\left[\left(1-\exp \left(-\langle\Delta t\rangle / t_{v}\right)\right) A_{i}^{n-1}+\left(\exp \left(-\langle\Delta t\rangle / t_{v}\right)\right.\right. \\
& \left.\left.-\exp \left(-2\langle\Delta t\rangle / t_{v}\right)\right) A_{i}^{n-2}\right]
\end{aligned}
$$

with $\langle\Delta t\rangle$ the average inter-event time. An even more general model could be derived by extending with more terms, but the range of two inter-event times is sufficient for the problems studied in this paper. The initial conditions for this recursive formula are given by $A_{i}^{i+1}=1$ based on the definition of $A_{i}^{n}$, and by $A_{i}^{i}=-1 /\left[1-\gamma E_{v} / E_{0}(1-\right.$ $\left.\left.\exp \left(\langle\Delta t\rangle / t_{v}\right)\right)\right]$, which is deduced from the stress concentration creation during slip event $i$ and the assumption that there was no stress concentration before. While all $A_{i}^{n}$ for $n>i$ are positive, this initial value $A_{i}^{i}$ is negative. The reason for this particularity lies in the difference of the slip event's effect on the stress concentration. The initial concentration is zero before slip event $i$ and is created during slip. Therefore its amplitude decreases until the next slip event $i+1$ (see peak at 0.32 in Fig. 4). However, all stress concentrations that have been created before are erased by every succeeding slip event and reappear in between (see peaks at 0.26 and at 0.29 in Fig. 4). This difference can also be observed in Fig. 7(a). A stress concentration is created at $0.218 \mathrm{~s}$ and reduces until $0.23 \mathrm{~s}$. At $0.233 \mathrm{~s}$, it is erased by the propagation of a slip event, after which it reappears until $0.25 \mathrm{~s}$. This difference between the first 

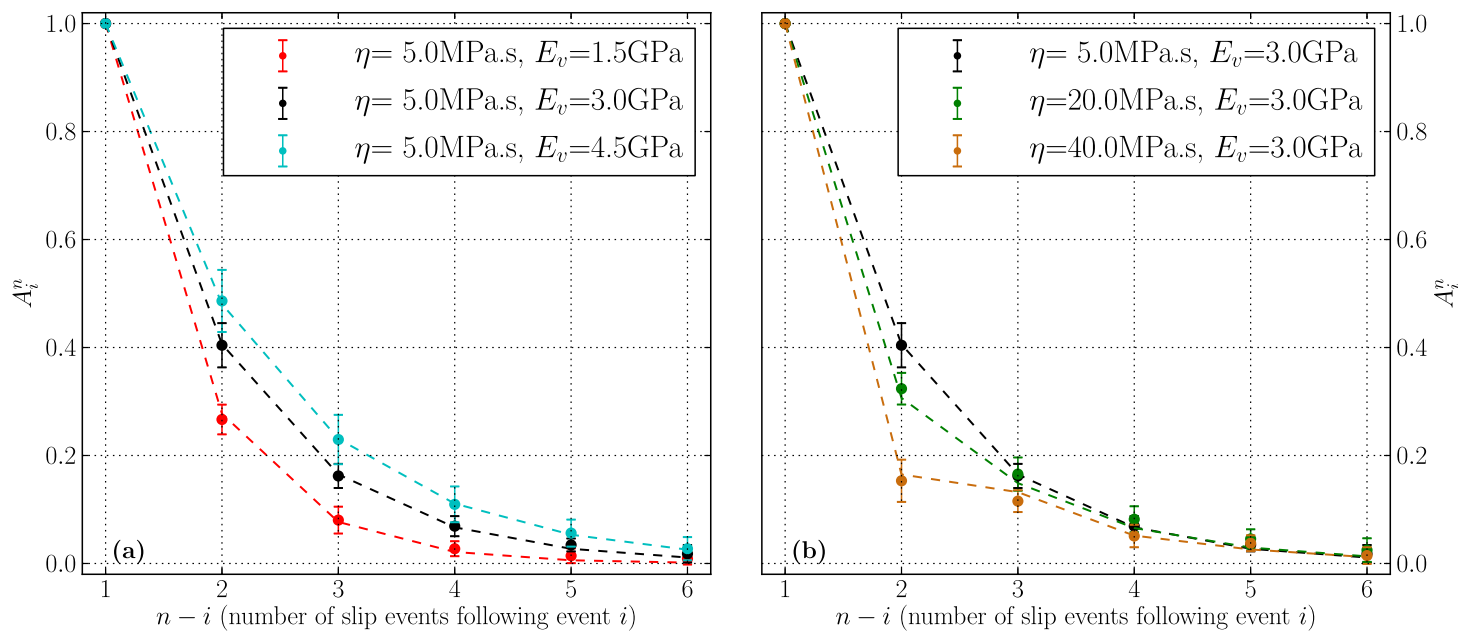

Fig. 8. Evolution of $A_{i}^{n}$ for different values of $E_{v}$ and $\eta$. Each selection of points represents the average behavior for all the positions $x_{i}$, with standard deviation. The black data is the reference case, as shown in Fig. 6. (a) $E_{v}$ is varied. A faster decrease rate is observed for smaller values of $E_{v}$. Dashed lines are the predictions based on Eq. (9). (b) $\eta$ is varied. Larger $\eta$ have faster decrease rates for $n-i<2$ and slower rates for $n-i>2$, indicating that the system does not have enough time to relax between two slip events. Dashed lines are the predictions based on Eq. (10).

viscous effect and all the following ones is also the reason for faster decrease rates for $n-i<2$ in Fig. 8(b). The decreasing viscous effect due to the creation of the stress concentration at $t=t_{i}$ has not been completely relieved at $t=t_{i+1}$ when the next slip event propagates and has therefore still a diminishing influence on $A_{i}^{i+2}$.

The proposed extension to complete relaxation within two inter-event times results in a good prediction of the observed behavior (see dashed lines in Fig. 8(b)).

\section{Influence of bimaterial interfaces}

Precursory slip in experimental setups are generated on non-symmetric frictional interfaces. In Rubinstein et al. (2007), the upper plate (slider) is thin, compared to the wider bottom plate (base), which has a higher rigidity. In the reference case, we consider a rigid base with infinite rigidity, which is thus an approximation made to the real cases where the rigidity contrast is not infinite. We investigate interfacial contrasts by considering three different cases: $(A)$ the base is rigid (reference case presented in Sections 3 and 4, (B) the base is deformable in plane-strain approximation, and $(C)$ the base is deformable in planestress approximation. In each case, the slider is always deformable, in plane-stress approximation. We analyze the existence, amplitude and decrease rate of the stress concentrations created by precursory slip.

First, we note that the number of precursor events varies depending on the characteristics of the bottom plate. For the three cases presented here, respectively 18, 12 and 9 precursors are observed before global sliding. Due to the differences in the rigidity of the base, the friction traction and frictional strength at the interface vary depending on the cases. Fig. 9(a) shows the shear traction at the interface after equilibrium of the normal load, before initiation of the shear loading. It reveals the differences in Poisson's expansion for different stiffness of the base. Also, we have observed that the contact pressure is lower in the corners when the lower plate is stiffer, and this could explain in particular why slip events are easier to initiate when the lower plate is stiffer.

The stress concentrations $A_{i}^{n}$ have a faster decrease rate when the bottom plate is deformable compared to the case where it is rigid (see Fig. 10). The difference of about 10\% can be accounted for by considering a different coefficient $\gamma$ when the bottom plate is deformable. A best fit gives a value of $\gamma=0.6$ ( $\gamma=0.608$ for plane-strain and $\gamma=0.628$ for plane-stress). No significant variation in the decrease rate is observed between the plane-strain and plane-stress cases.

To understand the origin of this difference, the time evolution of $\tau$ is analyzed at a particular position $x_{i}$ corresponding to a maximum of stress concentration (Fig. 7(a)-(c)). For the three cases, almost the same position of the interface is analyzed (the differences in position, of less than $2 \mathrm{~mm}$, are due to slightly different arrest locations). We focus on the initial shear traction level $\tau\left(x_{i}, t_{i}^{-}\right)$, before the creation of the stress concentration in $x_{i}$ (red dots in Fig. 7). In the reference case (rigid base), $\tau\left(x_{i}, t_{i}^{-}\right)$is lower than for deformable bases. This can also be seen in Fig. 9(b) (zoom) which shows the shear traction along the interface just before the propagation of the slip event that will stop at the positions marked by the vertical dashed lines $(x / L \approx 0.3$, position studied in Fig. 7). For the reference case, we can observe the lower value of $\tau$ at the arrest position of the next event (marked by vertical dashed lines). During the creation of the stress concentration in $x_{i}$, the shear traction will jump from $\tau\left(x_{i}, t_{i}^{-}\right)$to $\tau\left(x_{i}, t_{i}^{+}\right)$(from red to blue dots in Fig. 7). $\tau\left(x_{i}, t_{i}^{+}\right)$is the value of $\tau$ at the time of slip arrest, before relaxation starts. This jump from $\tau\left(x_{i}, t_{i}^{-}\right)$to $\tau\left(x_{i}, t_{i}^{+}\right)$will increase for lower initial stresses.

Fig. 10(b) reveals a correlation between the initial jump $\tau\left(x_{i}, t_{i}^{+}\right)-\tau\left(x_{i}, t_{i}^{-}\right)$and the value of $\gamma$ estimated at each position $x_{i}$. For a rigid base (dots), the values of $\tau\left(x_{i}, t_{i}^{+}\right)-\tau\left(x_{i}, t_{i}^{-}\right)$are between 1 and $1.5 \mathrm{MN}$, whereas when the base is deformable, this ratio is between 1.4 

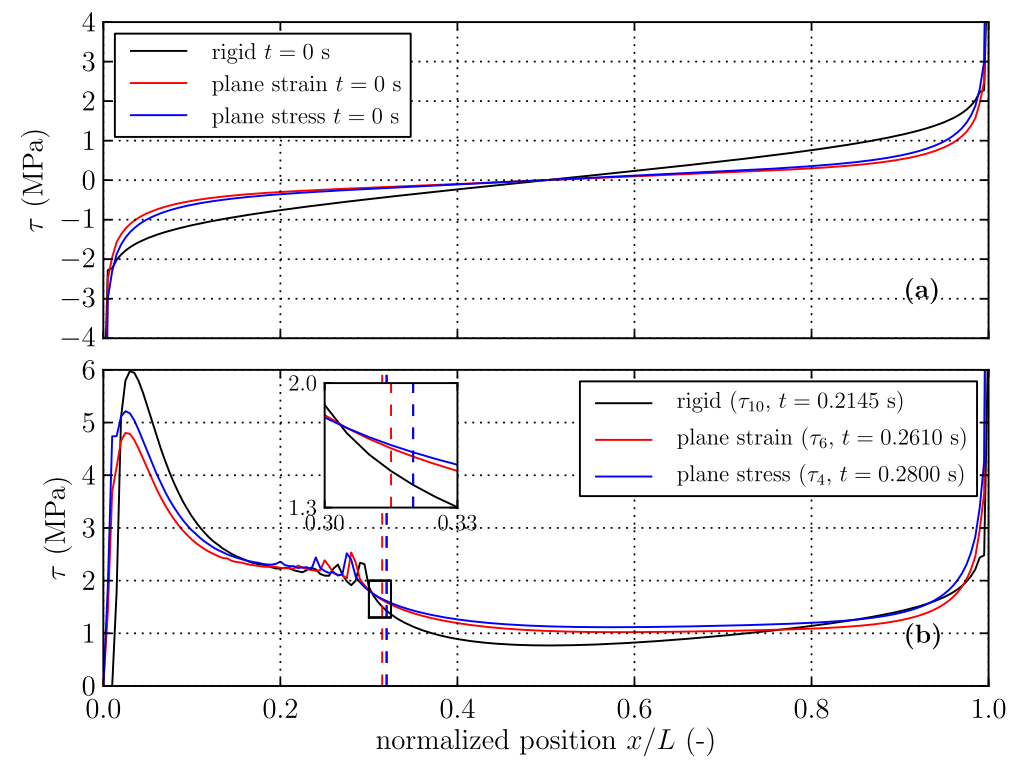

Fig. 9. $\tau$ along the interface at different times for the three different configurations of the base (a) At equilibrium, before the initiation of the shear load $(t=0)$. The stress profile results from the frustrated poisson's expansion. (b) Just before the initiation of the precursor $i$ stopping around $x / L=0.3$. The precursors indexes, and times, The time, different for each configuration, are indicated in the legend. The arrest location for each precursor is marked by the vertical dashed line (the black line below the blue, because the precursors have the same arrest position). At this position, the solid black line is located under the solid red and blue lines. $\tau\left(x_{i}, t_{i}^{-}\right)$will thus be smaller for a rigid base than for a deformable base. (For interpretation of the references to colour in this figure caption, the reader is referred to the web version of this article.)
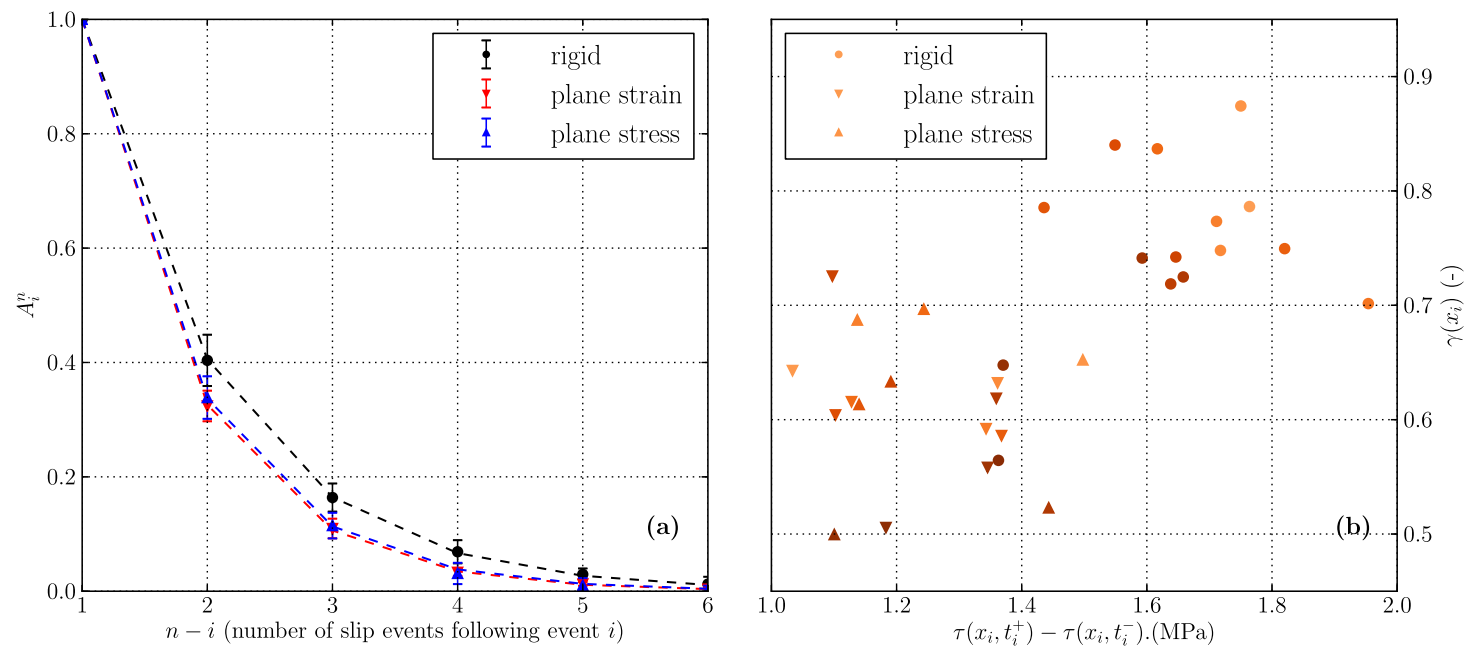

Fig. 10. (a) Evolution of the normalized amplitude of stress concentrations in a deformable-deformable interface (red and blue curves), compared with the reference rigid base (black line). Dots with error bars are the data from the simulations, and the dashed lines are the prediction using Eq. (9), and different $\gamma$ values. (b) Local values of $\gamma$ for all positions $x_{i}$ on the interface, as a function of the initial jump in shear stress $\tau\left(x_{i}, t_{i}^{+}\right)-\tau\left(x_{i}, t_{i}^{-}\right)$. Colors are function of the position $x_{i}$, as in Fig. 2, and symbols represent variation in the stiffness of the base. $\tau\left(x_{i}, t_{i}^{+}\right)-\tau\left(x_{i}, t_{i}^{-}\right)$corresponds to the difference between the blue and red dots in Fig. 7. (For interpretation of the references to colour in this figure caption, the reader is referred to the web version of this article.)

and $2 \mathrm{MN}$. The mean values of $\gamma$ estimated before represent well the mean behavior for a deformable $(\gamma=0.6)$ or a rigid base $(\gamma=0.758)$.

The amplitude of this jump will control the viscous relaxation occurring later on, and in particular the values of the shear stress before the succeeding events $\left(\tau_{i+1}\right.$ and following). The lower $\tau\left(x_{i}, t_{i}^{-}\right)$is, the lower will be the shear stress before the succeeding events, and the higher will be the jump to reach the frictional strength in each case. This will thus influence the coefficient $\gamma$ introduced before, which accounts for the time history of the drop.

Other effects could explain the differences between deformable-rigid and deformable-deformable interfaces. In particular, variations in $\sigma$ during slip are larger when 
the base is rigid, and can thus lead to larger variations in the frictional strength during slip events.

\section{Conclusion}

In this paper, we model sequences of precursory slip on an interface between two solids. At the tip of each arrested rupture, a stress concentration is created. When the following rupture propagates through that position, the stress concentration is erased. We show that while the interface is sticking during slip events, the erased stress concentrations reappear, and survive the propagation of several ruptures. At a given position, the amplitude of the stress concentration is decreasing with successive ruptures. We propose a theoretical model to describe how the stress concentration decreases between slip events, accounting for the bulk viscoelastic relaxation. This model describes the decrease rate of the stress concentration as the ratio of the viscous over the instantaneous Young's moduli. By varying the materials properties in the simulation, we show that the analytical model proposed describes well the average decrease rate of stress concentrations, if the relaxation time is short enough so that the system has sufficient time to fully relax between two slip events.

In the case where complete relaxation does not have time to occur between two slip events, a second slip event will propagate on an interface that is not at equilibrium. We extend the previous analytical model and proposed a new evolution of $A_{i}^{n}$, which takes into account the history of two past events, and not only of the previous one. The formulation could be extended to account for more than two events. This new analytical prediction appropriately describes the decrease rate of stress concentrations in our simulations when the relaxation period is longer than the inter-event time.

Finally, the effect of bilateral interfaces on the survival of stress concentrations has been investigated. We show that when the bottom plate is deformable, the decrease rate of stress concentrations is faster compared to a rigid bottom plate, and the variation is of the order of $10 \%$. We suggest that the observed difference is due to variations in initial stresses created by different stiffnesses of the base, and propose a different coefficient $\gamma$ to model the behavior on deformable-deformable interfaces. No significant variation is observed between the plane-stress or plane-strain approximation.

The comparison between deformable-rigid and deformable-deformable interfaces reveal some differences in the global and local behavior of the system. These observations reveal the importance of investigating in more details the influence of non-symmetrical interfaces, and quantify the errors made when considering a rigid body on one side of the interface. In particular, the influence of non-symmetrical interfaces on rupture initiation and propagation could be investigated in more details.

\section{Appendix A. Model for evolution of $\tau$}

The time evolution of $\tau$ due to the propagation of a slip event is considered as the contribution of 3 terms: (1) the drop in $\tau$ due to the slip, (2) the relaxation of the viscoelastic material and (3) a term accounting for the shear loading. From Eq. (5), we derive the evolution of $\tau$ for an instantaneous (negative) drop $\Delta \tau_{n}=\tau_{n}^{+}-\tau_{n}^{-}$, where $\tau_{n}^{-}$ and $\tau_{n}^{+}$are respectively the interface shear stresses just before and after the slip event occurring at time $t_{n}$. The notation with superscript - and + for before and after a slip event is hereafter also applied to other terms.

We assume an initial state of equilibrium. In the planestress case, $\Delta \tau_{n}$ corresponds to $\left[-E_{0} /(1+v)\right] \Delta \epsilon_{x y}$ in Eq. (5), and $\tau\left(x, t_{n}^{-}\right)$to the initial state. We introduce a loading term $p_{\tau}$, assumed to be constant with time and only varying with the position. We thus have the following evolution for $t \geqslant t_{n}$ :

$$
\begin{aligned}
\tau(x, t)= & \tau\left(x, t_{n}^{-}\right)-\frac{\Delta \tau_{n}(x)}{E_{0}}\left[E_{\infty}+E_{v} \exp \left(-\frac{t-t_{n}}{t_{v}}\right)\right] \\
& +p_{\tau}(x)\left(t-t_{n}\right)
\end{aligned}
$$

Considering that $\tau\left(x, t_{n}^{-}\right)=\tau\left(x, t_{n}^{+}\right)-\Delta \tau_{n}(x)$ and that $E_{0}=E_{\infty}+E_{v}$, we have:

$$
\begin{aligned}
\tau(x, t)= & \tau\left(x, t_{n}^{+}\right)+\Delta \tau_{n}(x) \frac{E_{v}}{E_{0}}\left[1-\exp \left(-\frac{t-t_{n}}{t_{v}}\right)\right] \\
& +p_{\tau}(x)\left(t-t_{n}\right)
\end{aligned}
$$

where the first term is the shear stress just after a slip event, the second term corresponds to the time dependent viscous relaxation, and the third term to the shear loading.

Assuming large enough local slip at the interface, satisfying $l>d_{c}$, the shear stress just after the propagation of the slip event $n$ is:

$\tau\left(x, t_{n}^{+}\right)=\mu_{k} \sigma\left(x, t_{n}^{+}\right)$.

The stress drop can therefore be described by

$\Delta \tau_{n}(x)=\gamma\left[\tau\left(x, t_{n}^{+}\right)-\tau\left(x, t_{n}^{-}\right)\right]=\gamma\left[\mu_{k} \sigma\left(x, t_{n}^{+}\right)-\tau\left(x, t_{n}^{-}\right)\right]$

where the coefficient $\gamma(0<\gamma<1)$ is introduced to "smoothen" the drop in order to take into account that the stress drop is not instantaneous.

Neglecting volume changes due to $\operatorname{slip}\left(\Delta \epsilon_{k k}=0\right.$ in Eq. (3)), $\sigma$ does not change during a slip event and we can approximate that $\sigma\left(x, t_{n}^{-}\right)=\sigma\left(x, t_{n}^{+}\right)$. Thus, we can simplify Eq. (A.3) to

$\Delta \tau_{n}(x)=\gamma\left[\mu_{k} \sigma\left(x, t_{n}^{-}\right)-\tau\left(x, t_{n}^{-}\right)\right]$

and describe the evolution of the contact pressure

$\sigma_{n+1}(x)=\sigma_{n}(x)+p_{\sigma}(x)\left(t_{n+1}-t_{n}\right)$

which depends only on the loading $p_{\sigma}$. We simplified the notation by setting $\sigma_{n}(x)=\sigma\left(x, t_{n}^{-}\right)$, which we also do in the following for $\tau_{n}(x)=\tau\left(x, t_{n}^{-}\right)$.

Combining everything together, we can now express the shear stress just before slip event $n+1$ as a function of the stresses before the previous rupture and Eq. (A.2) becomes:

$$
\begin{aligned}
\tau_{n+1}(x)= & \mu_{k} \sigma_{n}(x)+\gamma \frac{E_{v}}{E_{0}} B_{n}\left(t_{n+1}\right)\left[\tau_{n}(x)-\mu_{k} \sigma_{n}(x)\right] \\
& +p_{\tau}(x)\left(t_{n+1}-t_{n}\right)
\end{aligned}
$$


with $B_{n}(t)=1-\exp \left(-\frac{t-t_{n}}{t_{v}}\right)$.

\section{Appendix B. Simplified stress concentration evolution}

Assuming complete relaxation between slip events, the shear stress is described by Eq. (A.4) with $B_{n}\left(t_{n+1}\right) \approx 1$, and following Eq. (6), the stress concentration can be expressed as:

$$
\begin{aligned}
\Delta_{i}\left(\tau_{n+1}\right)-\Delta_{i}\left(\tau_{\text {ref }}\right)= & \gamma \frac{E_{v}}{E_{0}}\left[\Delta_{i}\left(\tau_{n}\right)-\Delta_{i}\left(\tau_{\text {ref }-1}\right)\right] \\
& +\left(t_{n+1}-t_{n}-t_{\text {ref }}+t_{\text {ref }-1}\right)\left(p_{\tau}\left(x_{i}\right)-p_{\tau}\left(x_{i}^{\prime}\right)\right) \\
& +\mu_{k}\left(1-\gamma \frac{E_{v}}{E_{0}}\right)\left[\sigma_{n}\left(x_{i}\right)-\sigma_{n}\left(x_{i}^{\prime}\right)+\sigma_{\text {ref }-1}\left(x_{i}\right)\right. \\
& \left.-\sigma_{\text {ref }-1}\left(x_{i}^{\prime}\right)\right]
\end{aligned}
$$

Since the positions $x_{i}$ and $x_{i}^{\prime}$ are very close, we can approximate $\sigma_{n}\left(x_{i}\right) \approx \sigma_{n}\left(x_{i}^{\prime}\right)$ (see Fig. 5(d)). Furthermore, our simulations present, for a given configuration, a precursor frequency that is approximately constant over the entire time. Therefore we can approximate $t_{\text {ref }}-t_{\text {ref }-1}$ $\approx t_{n+1}-t_{n}$. Moreover, the reference stress concentration was defined as when its changes are negligible between two slip events, which enables us to set $\Delta_{i}\left(\tau_{\text {ref }}\right) \approx \Delta_{i}\left(\tau_{\text {ref-1 }}\right)$. Following these assumptions, Eq. (B.1) becomes:

$\Delta_{i}\left(\tau_{n+1}\right)-\Delta_{i}\left(\tau_{\text {ref }}\right)=\gamma \frac{E_{v}}{E_{0}}\left[\Delta_{i}\left(\tau_{n}\right)-\Delta_{i}\left(\tau_{\text {ref }}\right)\right]$,

which leads to

$A_{i}^{n+1}=\gamma \frac{E_{v}}{E_{0}} A_{i}^{n} \quad$ for $n>i+1$.

\section{Appendix C. Stress concentration evolution}

We propose a theoretical model that is more general than the simplified version described in Appendix B. Instead of imposing that complete relaxation has to occur during a period of a single inter-event time, we assume that relaxation is fully achieved within the period of two inter-event times. In order to keep it as simple as possible, we assume that the reference stress state is constant at the location of the stress concentration, which is correct for the large majority of slip events. The normalized amplitude of stress concentrations can, therefore, be reduced to $A_{i}^{n}=\Delta_{i}\left(\tau_{n}\right) / \Delta_{i}\left(\tau_{i+1}\right)$. The evolution of the shear stress is given by Eq. (A.1), which is simplified to

$$
\begin{aligned}
\tau_{n}(x, t)= & \tau_{n-1}(x) \\
& +\left[\tau_{n-1}(x)-\mu_{k} \sigma_{n-1}(x)\right]\left(\gamma \frac{E_{v}}{E_{0}} B_{n-1}(t)-1\right)
\end{aligned}
$$

with $B_{n}(t)=\left[1-\exp \left(-\left(t-t_{n}\right) / t_{v}\right)\right]$. The difference between the maximal and minimal stress of a given stress concentration can be expressed as a function of the stress concentration before the previous slip event by

$\Delta_{i}\left(\tau_{n}(t)\right)=\gamma \frac{E_{v}}{E_{0}} B_{n-1}(t) \Delta_{i}\left(\tau_{n-1}\right)$ for $t \geqslant t_{n-1}$ for any time after slip event $n-1$ and before slip event $n$.

If we consider that the stress concentration is still affected by the viscous relaxation of the slip event $n-2$, we can sum Eq. (C.2) to

$$
\begin{aligned}
\Delta_{i}\left(\tau_{n}(t)\right)= & \gamma \frac{E_{v}}{E_{0}}\left[B_{n-1}(t) \Delta_{i}\left(\tau_{n-1}\right)+\left(B_{n-2}(t)\right.\right. \\
& \left.\left.-B_{n-2}\left(t_{n-1}\right)\right) \Delta_{i}\left(\tau_{n-2}\right)\right] \text { for } t \geqslant t_{n-1} .
\end{aligned}
$$

The term $\gamma E_{v} / E_{0} B_{n-2}\left(t_{n-1}\right) \Delta_{i}\left(\tau_{n-2}\right)$ is subtracted in Eq. (C.3) in order to satisfy the condition that $\Delta_{i}\left(\tau_{n}\left(t_{n-1}\right)\right)=0$, because just after the slip event $n-1$ passed, there was no stress concentration.

Considering the stress concentration just before slip event $n$, we can write $t=t_{n}, t_{n}-t_{n-1}=\Delta t$ and $t_{n}-t_{n-2}$ $=2 \Delta t$. Therefore, dividing Eq. (C.3) by $\Delta_{i}\left(\tau_{i+1}\right)$, we find the relation describing the evolution of

$$
\begin{aligned}
A_{i}^{n}= & \gamma \frac{E_{v}}{E_{0}}\left[1-\exp \left(-\Delta t / t_{v}\right)\right] A_{i}^{n-1} \\
& +\gamma \frac{E_{v}}{E_{0}}\left[\exp \left(-\Delta t / t_{v}\right)-\exp \left(-2 \Delta t / t_{v}\right)\right] A_{i}^{n-2}
\end{aligned}
$$

for any given stress concentration. Any recursive formula that takes into account two past values needs two initial conditions. The first one is here given by

$A_{i}^{i+1}=1$

based on the initial definition of the normalized stress concentration. Considering that the stress concentration was created during slip event $i$ (where no concentration was before) and that it is reduced during the first inter-event period from $t_{i}$ to $t_{i+1}$, we can write $A_{i}^{i+1}=-\left(1-B_{i}\left(t_{i+1}\right)\right) A_{i}^{i}$. This leads, using the first initial condition, to the second initial condition:

$A_{i}^{i}=-1 /\left[1-\gamma E_{v} / E_{0}\left(1-\exp \left(\Delta t / t_{v}\right)\right)\right]$

\section{References}

Akantu, 2013. <http://1sms.epfl.ch/akantu>

Amundsen, D., Scheibert, J., Thgersen, K., Trmborg, J., Malthe-Srenssen, A., 2012. 1d model of precursors to frictional stick-slip motion allowing for robust comparison with experiments. Tribol. Lett. 45, 357-369. http://dx.doi.org/10.1007/s11249-011-9894-3.

Andrews, D.J., 1999. Test of two methods for faulting in finite-difference calculations. Bull. Seismol. Soc. Am. 89, 931-937.

Bar-Sinai, Y., Spatschek, R., Brener, E.A., Bouchbinder, E., 2013. Instabilities at frictional interfaces: creep patches, nucleation and rupture fronts. arXiv, preprint arXiv:1306.3658.

Bartolomeo, M.D., Massi, F., Baillet, L., Culla, A., Fregolent, A., Berthier, Y., 2012. Wave and rupture propagation at frictional bimaterial sliding interfaces: From local to global dynamics, from stick-slip to continuous sliding. Tribol. Int. 52, 117-131. http://dx.doi.org/ 10.1016/j.triboint.2012.03.008.

Ben-David, O., Cohen, G., Fineberg, J., 2010. The dynamics of the onset of frictional slip. Science 330, 211-214.

Ben-David, O., Rubinstein, S.M., Fineberg, J., 2010. Slip-stick and the evolution of frictional strength. Nature 463, 76-79.

Ciccotti, M., Mulargia, F., 2004. Differences between static and dynamic elastic moduli of a typical seismogenic rock. Geophys. J. Int. 157, 474477. http://dx.doi.org/10.1111/j.1365-246X.2004.02213.x.

Cochard, A., Rice, J.R., 2000. Fault rupture between dissimilar materials: III-posedness, regularization, and slip-pulse response. J. Geophys. Res. 105, 25891-25907. http://dx.doi.org/10.1029/2000JB900230 <http:// dx.doi.org/10.1029/2000JB900230> .

Coker, D., Rosakis, A., Needleman, A., 2003. Dynamic crack growth along a polymer composite-homalite interface. J. Mech. Phys. Solids 51, 425460, dx.doi.org/10.1016/S0022-5096(02)00082-0. 
DeDontney, N., Rice, J.R., Dmowska, R., 2012. Finite element modeling of branched ruptures including off-fault plasticity. Bull. Seismol. Soc. Am. 102, 541-562. http://dx.doi.org/10.1785/0120110134.

Kammer, D.S., Yastrebov, V.A., Spijker, P., Molinari, J.F., 2012. On the propagation of slip fronts at frictional interfaces. Tribol. Lett. 48, 27 32, 0.1007/s11249-012-9920-0.

Kammer, D.S., Yastrebov, V.A., Anciaux, G., Molinari, J.F., 2014. The existence of a critical length scale in regularised friction. J. Mech. Phys. Solids 63, 40-50, dx.doi.org/10.1016/j.jmps.2013.10.007.

Lykotrafitis, G., Rosakis, A., 2006. Dynamic sliding of frictionally held bimaterial interfaces subjected to impact shear loading. Proc. R. Soc London, Ser. A 462, 2997-3026. http://dx.doi.org/10.1098/ rspa.2006.1703.

Maegawa, S., Suzuki, A., Nakano, K., 2010. Precursors of global slip in a longitudinal line contact under non-uniform normal loading. Tribol. Lett. 38, 313-323. http://dx.doi.org/10.1007/s11249-010-9611-7.

Otsuki, M., Matsukawa, H., 2013. Systematic breakdown of amontons' law of friction for an elastic object locally obeying amontons' law. Sci. Rep. 3,1586
Prakash, V., Clifton, R., 1993. Time resolved dynamic friction measurements in pressure-shear. In: Ramesh, A.K.T. (Ed.), Experimental Techniques in the Dynamics of Deformable Solids, vol. 165. Am. Soc. of Mechanical Eng., New-York, pp. 33-48.

Radiguet, M., Kammer, D.S., Gillet, P., Molinari, J.F., 2013. Survival of heterogeneous stress distributions created by precursory slip at frictional interfaces. Phys. Rev. Lett. 111, 164302. http://dx.doi.org/ 10.1103/PhysRevLett. 111.164302.

Rubinstein, S.M., Cohen, G., Fineberg, J., 2004. Detachment fronts and the onset of dynamic friction. Nature 430, 1005-1009.

Rubinstein, S.M., Cohen, G., Fineberg, J., 2007. Dynamics of precursors to frictional sliding. Phys. Rev. Lett. 98, 226103. http://dx.doi.org/ 10.1103/PhysRevLett. 98.226103.

Trømborg, J., Scheibert, J., Amundsen, D.S., Thgersen, K., Malthe-Srenssen, A., 2011. Transition from static to kinetic friction: Insights from a $2 \mathrm{~d}$ model. Phys. Rev. Lett. 107, 074301. http://dx.doi.org/10.1103/ PhysRevLett. 107.074301.

Zener, C., 1948. Elasticity and Anelasticity of Metals. University of Chicago Press. 